\title{
Health Perception and Health Behaviors of Elder Tibetans Living in India and Switzerland
}

\author{
Tenzin Wangmo
}

Published online: 6 September 2011

(C) Springer Science+Business Media, LLC 2011

\begin{abstract}
Tibetan elders have lived in exile since the failed uprising against Chinese rule in 1959. Using mixed methods approach, this study explored the perceived health and health behaviors of 30 older Tibetans living in India and Switzerland using Bronfenbrenner's Ecological Systems Model. Results indicate that elder Tibetans living in Switzerland enjoyed better health and well-being than those living in India. Because of the availability of old age benefits, pensions, and health insurance, participants in Switzerland had greater financial independence, and fewer problems with healthcare access and affordability than those in India. In addition to financial reimbursement, the contribution and importance of personal, familial, community, and religious factors to participant's health behaviors are examined. The study is a case example depicting the impact of different factors from micro to macro on the health and well-being of a refugee group. Its implications such as the implementation of old age benefits for older persons living in developing countries are discussed.
\end{abstract}

Keywords Health behaviors · Well-being · Elder Tibetans · Older refugees ·

Ecological systems model

\section{Introduction}

This paper explores and compares the perceived health and health behaviors of older Tibetan refugees who have lived in India and Switzerland since the failed Tibetan upraising against Chinese rule in 1959. Studies exploring health and health behaviors of elder Tibetans are lacking. Available research concerning health of Tibetans in diaspora describe their physical and psychological health (Bera 2004; Dolma et al. 2006; Holtz 1998; Mills et

T. Wangmo $(\triangle)$

Institute for Biomedical Ethics, University of Basel, Missionsstrasse 24, Basel 4055, Switzerland e-mail: tenzin.wangmo@unibas.ch

T. Wangmo

e-mail: tenwangmo@yahoo.com 
al. 2005). Many studies have explored the diasporic Tibetan identity (Anand 2000; Houston and Wright 2003; Kleiger 1992; Korom 1999; Mountcastle 1997; Routray 2007) but a very few have examined the aging experience of older Tibetans in Tibet and in diaspora (Goldstein and Beall 1997; Wangmo and Teaster 2010; Wangmo 2010).

Goldstein and Beall (1997) characterized traditional patriarchal Tibetan society where parents devoted their time to the family and to the upbringing of their children. However when children became adults, they took over the role of care providers and parents dedicated their old age to religious activities (Childs et al. 2011; Goldstein and Beall 1997). Wangmo and Teaster (2010) discussed aging experiences of 14 elder Tibetans in India and their engagement in religious activities. They found that for older Tibetans, participation in religious activities collects good karma in order to influence their next life, and this engagement becomes an important part of their old age. This study also reported that these elders received financial support from their children, thereby contributing to the elders' well-being. Another study explored intergenerational relationship between Tibetan elders and their children in India and Switzerland (Wangmo 2010). Study results revealed the significance of financial support from children for elders living in India and emotional support for those living in Switzerland.

\section{Health of Tibetan refugees}

The overall health of a refugee is influenced by factors such as migration, losses incurred in the home country, traumatic events encountered, and hardships survived during the transition including stays in refugee camps (Adams et al. 2004; Keyes and Kane 2004). Mental and physical health problems may present as a result of past experiences and are prevalent among refugees. These include conditions such as post traumatic stress disorder (PTSD), anxiety, depression, and infectious diseases (Burgess 2004; Joeffe et al. 2003; Smith 2003; Wrobel et al. 2009).

Similarly, literature on the health of Tibetans living in diaspora has documented PTSD, depression, and anxiety among Tibetan refugees in India and Nepal due to traumatic events suffered in Tibet and the ordeal of escaping through the Himalayas (Dolma et al. 2006; Mills et al. 2005). Holtz (1998) assessed the prevalence of mental disorders among 35 refugee nuns and laypersons arrested and tortured in Tibet as well as 35 control participants. Not surprisingly, the tortured survivors had significantly higher anxiety scores than the nontortured survivors.

Besides the psychological health of Tibetan refugees, their physical health has been examined. Bera (2004) found that Tibetans drank high quantities of buttered salt tea, especially older Tibetans, and that they consumed $12.5 \%$ more calories than the recommended dietary allowances. High amounts of fat and caloric consumption resulted in increasing obesity among Tibetans, concomitantly increasing their risk for other health problems. The most common ailments were digestive disorders, upper respiratory diseases, tuberculosis, arthritis, joint pain, and back pain.

Other studies exploring the health of Tibetans concluded that Tibetan refugees in India had a four times higher prevalence of tuberculosis than that of native Indians (Nelson et al. 2005). This higher prevalence is particularly concerning because Prost (2008) found inequalities related to diagnosis and disclosure of tuberculosis among Tibetans in India. Like Tibetans in India, those in the United States and Canada have a higher prevalence of tuberculosis, with more drug resistant strains than any other members of Asian populations who test positive for the disease (Lee et al. 2001; Marras et al. 2003). 
Despite disease burden and unique health behaviors, scholars have demonstrated that the health status of Tibetans is similar to that of residents of developed countries (Bhatia et al. 2002; Tripathy and Gupta 2005; Tripathy et al. 2006). Elder Tibetans now suffer from cardiovascular disease, obesity, and hypertension (Bhatia et al. 2002; Tripathy et al. 2006). By some measures, health status indicators of Tibetans are actually better than those of native members of their host country, as depicted by the higher birth weight of Tibetan babies compared to that of Indian babies (Tripathy and Gupta 2005; Tripathy et al. 2006).

Factors affecting health of older immigrants and refugees

Research concerning the health of older immigrants and refugees reveal the role of social support and religion on their health. The positive effect of social support on older immigrants' well-being has been widely reported (Ajrouch 2007; Diwan et al. 2004; Yeung and Fung 2007). Soonthornchaiya and Dancy (2006) found that adherence to Buddhism and to Buddhist teachings of acceptance of change helped older Thai immigrants cope with depression. Several studies have also established the positive health effects associated with religious activities among older Taiwanese, Japanese, and Palestinians (Chaaya et al. 2007; Hahn et al. 2004; Krause et al. 1999; Yeager et al. 2006).

Other important factors impacting the health of refugees and immigrants are trust issues and structural barriers to health. Feldman et al. (2007) stated that for refugees to seek proper and timely healthcare, positive relations based on trust needed to be built between refugees and their healthcare practitioners. Negative health behaviors were found among Russian immigrants who distrusted health information received from the media and healthcare providers (Benisovich and King 2003).

Wong et al. (2006) concluded that for Cambodian refugees in the United States, structural barriers such as the high cost of healthcare, poor access to healthcare, discrimination, and language problems were significant barriers to better health and health promotion. Similarly, immigrant and refugee women in Canada perceived lack of access to culturally, linguistically, and gender appropriate healthcare services as barriers to seeking healthcare (Fung and Wong 2007).

\section{Bronfenbrenner's ecological systems model}

The framework used for this inquiry is Bronfenbrenner's Ecological Systems Model (Bronfenbrenner 1977; Bronfenbrenner and Morris 1998). The model provides an understanding of how different factors affect health and health behaviors. It examines the interaction of four ecological systems on the development of an individual: the microsystem, the mesosystem, the exosystem, and the macrosystem. The microsystem is the relationship between the individual and his/her immediate environment. For this study it includes personal factors such as attitudes and behaviors. The mesosystem is the interconnection of microsystems, or the interrelations among major settings in which the person lives. The mesosystems examined consisted of the relationship between the elder and his or her family as well as how the family aids in the elder's understanding of his or her present circumstances. The exosystem is an extension of the mesosystem which includes both formal and informal institutions such as the community in which the elder resides and the healthcare system that he or she utilizes. Finally, the macrosystem represents overarching institutional patterns of the culture including Tibetan religion and the effects of the policies of the two countries-India and Switzerland - in maintaining health and wellbeing of older Tibetans. 


\section{Methods}

Study sites

India and Switzerland were chosen as the countries of examination because they were the first countries to offer refuge to exiled Tibetans (Corlin 1991; Ott-Marti 1976; Woodcock 1970) and are home to the oldest communities of Tibetan refugees living in a developing and a developed country, respectively. India is the largest democracy in the world with a population of 1.1 billion. In 1999, eight percent of India's one billion people were 60 years or older (Chakraborti 2004). Life expectancy of Indians at birth is 62.1 years for men and 63.7 years for women (Jamuna 2000). Although India has extensive healthcare systems, it does not have universal healthcare for its residents. Healthcare is mostly provided on a payment per service basis.

Unlike India, Switzerland is a federal republic with a population of 7.8 million (Federal Statistic Office 2011). In 2009, older adults made up $16.8 \%$ of the population. Life expectancy was 80.1 years for men and 84.5 years for women. Switzerland provides universal healthcare for its residents.

Two Tibetan communities in India and Switzerland were studied. In India, the Bylakuppe Tibetan settlement was chosen as the study site because it is home to the largest and oldest Tibetan community in India. Bylakuppe is an agricultural settlement and is subdivided into 23 villages. Tibetan refugees were first resettled from India to Switzerland in 1961 (Corlin 1991; Ott-Marti 1976). Tibetans who resettled from the 1960s through 1970s typically live in German speaking cantons. Participants were recruited from two German speaking cantons, namely, St. Gallen and Zurich.

\section{Study participants}

Thirty older Tibetans participated in this study, 16 from India and 14 from Switzerland (Table 1). Of these, there were 12 women and 18 men, with ages ranging from 60 to 92 years. All fled Tibet after the failed uprising against the Chinese occupation in 1959.

\section{Recruitment}

In both locations, local informants introduced me to potential participants and informed them about the study. This protocol was followed to ensure participants' ease in communicating with me when I approached them about the study at a later time. After the initial contact, I explained the purpose of the study and inquired as to whether they would be willing to participate. If the elder was interested in participating, an interview appointment at a time and place of their choosing was scheduled. Participants were asked if they knew of other elder Tibetans who might be interested in participating. Thus, a participant pool was built upon the suggestions of others, a hallmark of the snowball sampling (Cresswell 2003).

Study methods

The study methods were approved by the Institutional Review Board of the University of Kentucky. The study used a qualitative methodology (Bernard 2002; Johnson 2002). Interview questions solicited information about how the participants have developed healthcare behaviors among the society in which they live as well as the impact of family, 
Table 1 Participant Demography (2007-2008)

\begin{tabular}{|c|c|c|c|c|c|}
\hline Participant & Gender & Age & Marital status & $\begin{array}{l}\text { Number } \\
\text { of children }\end{array}$ & Country \\
\hline Penpa & $\mathrm{F}$ & 79 & Married & 1 & 1 \\
\hline Gyaltsen & $\mathrm{M}$ & 92 & Widowed & 3 & 1 \\
\hline Dhondup & $\mathrm{M}$ & 74 & Married & 4 & 1 \\
\hline Mingmar & $\mathrm{M}$ & 75 & Married & 0 & 1 \\
\hline Nyendak & $\mathrm{M}$ & 73 & Married & 1 & 1 \\
\hline Lhakpa & M & 65 & Married & 7 & 1 \\
\hline Tsomo & $\mathrm{F}$ & 60 & Married & 7 & 1 \\
\hline Tashi & M & 64 & Never Married & 0 & 1 \\
\hline Gyalpo & $\mathrm{M}$ & 76 & Never Married & 0 & 1 \\
\hline Phurbu & $\mathrm{M}$ & 73 & Married & 0 & 1 \\
\hline Tsering & $\mathrm{F}$ & 78 & Married & 5 & 1 \\
\hline Dawa & M & 79 & Widowed & 4 & 1 \\
\hline Passang & $\mathrm{F}$ & 73 & Married & 4 & 1 \\
\hline Dolma & $\mathrm{F}$ & 75 & Widowed & 1 & 1 \\
\hline Ngodup & M & 77 & Married & 5 & 1 \\
\hline Nyima & $\mathrm{F}$ & 84 & Widowed & 5 & 1 \\
\hline Yeshi & M & 72 & Widowed & 4 & 2 \\
\hline Yangchen & $\mathrm{F}$ & 63 & Widowed & 4 & 2 \\
\hline Youdon & $\mathrm{F}$ & 63 & Married & 0 & 2 \\
\hline Tsewang & $\mathrm{M}$ & 62 & Married & 5 & 2 \\
\hline Pema & $\mathrm{F}$ & 69 & Divorced & 4 & 2 \\
\hline Namgyal & $\mathrm{M}$ & 67 & Married & 3 & 2 \\
\hline Sonam & M & 79 & Married & 1 & 2 \\
\hline Tenpa & $\mathrm{M}$ & 67 & Never Married & 0 & 2 \\
\hline Wangchuk & M & 70 & Widowed & 3 & 2 \\
\hline Topgyal & $\mathrm{M}$ & 72 & Widowed & 4 & 2 \\
\hline Sangmo & $\mathrm{F}$ & 70 & Widowed & 6 & 2 \\
\hline Tseten & $\mathrm{M}$ & 73 & Widowed & 4 & 2 \\
\hline Dolkar & $\mathrm{F}$ & 75 & Widowed & 5 & 2 \\
\hline Kyizom & $\mathrm{F}$ & 85 & Widowed & 7 & 2 \\
\hline
\end{tabular}

All names are pseudonyms; $M$ male; $F$ female; $1=$ India; $2=$ Switzerland

community, the healthcare system, religion, and governmental policies on their perceived health and healthcare behaviors.

In addition, two structured surveys were used to gather data on the elders' perceived health. The first was the Short Form Health Survey (SF-12), a self-report of participant health status and well-being with 12 questions examining physical and mental health (Ware et al. 1996). The SF-12 is widely used health measure with reliable internal consistency and discriminant validity (Resnick and Nahm 2001; Ware et al. 1996).

A second instrument was the Activities of Daily Living (ADL) and Instrumental Activities of Daily Living (IADL) used to measure the functional ability of the participants (Lawton and Brody 1969). These scales have been widely used to assess functional capacity of older adults. 
Translation The interview guide and SF-12 were translated into Tibetan. I translated the surveys to Tibetan in collaboration with one other bilingual Tibetan. I also backtranslated (i.e., translated the Tibetan version of the measure back to English) to check for consistency and transferability of meanings during translation. The ADL and the IADL questionnaires were not translated beforehand because the questions are simple and succinct. Instead, I translated ADL and IADL measures for the elders during the interview.

Most participants were not literate, and so written consent was not obtained. Participants orally consented to this study with their expressed willingness to talk to me serving as their informed consent. All interviews were conducted in participants' homes and they lasted from 1 to $3 \mathrm{~h}$ in duration. These interviews were tape recorded upon consent of the participant in order to ensure accuracy of the data for final analysis. I entered the tape recorded interviews and field notes verbatim from Tibetan to English.

Analysis

Data obtained from the SF-12, ADL, and IADL were entered into a spreadsheet, and double-checked for errors. The SF-12 was scored using a five point Likert scale. Scores 1-5 were recoded into values ranging from 0 to 100 using Litwin's (2002) score conversion scheme. Six questions each denoting physical health and mental health were grouped together, and the mean score for these questions were calculated. Mean scores can range from 0 to 100 , with a higher score representing the best possible health.

The ADL and IADL scales were scored as " 1 " indicating an elder's ability to perform the specific function, and " 0 " to denote the elder having difficulty with the activity.

Field notes were compared with the transcriptions to ensure that all data were taken into consideration for analysis. Transcribed interviews were analyzed using open and axial coding (Bernard 2002; Denzin and Lincoln 2000; Strauss and Corbin 1990). During open coding, transcribed data were broken down, compared, categorized, and conceptualized using line-by-line coding. These analyzed data were then sorted using axial coding, and then grouped into specific themes relating to context and conditions that influenced the health and health behaviors of the participants. To protect participant confidentiality, each participant was given a pseudonym.

\section{Limitations}

There are several limitations to the study. First, this study used a small convenience sample. Therefore, the study sample may not be representative of all older Tibetans living either in India or Switzerland. Second, social desirability may have affected the elders' responses. Elders may have felt the need to present the better parts of their experiences and opinions to a Tibetan student. Third, the scales utilized to measure participants' perception of health have not been used previously with older Tibetan samples. In addition, open-ended questions were not utilized to triangulate participants' perceived health status with the results obtained from the scales. Finally, it was difficult to administer the scale questions because of the multiple choice format. Participants were unsure about selecting an answer between good and fair or between very good and excellent. Therefore, the validity of the responses from these scales may be questionable. Despite its limitations, this study is the first one to empirically examine and compare the systematic effects of different factors on the perceived health and health behaviors of older Tibetans living in India and Switzerland. 


\section{Results}

The 30 participants have lived in two or more very different countries and have experienced various socioeconomic conditions in Tibet, India, and/or Switzerland. Study results are presented using Bronfenbrenner's Ecological Systems Model preceded by the results from the health surveys. The findings are organized below under the following themes: personal factors affecting health (microsystem), connection with family (mesosystem), community and healthcare (exosystem), and religion and government policies (macrosystem).

Findings from health surveys

Elders' perception of health was assessed using one question from the SF-12: "In general, would you say your health is__," with five possible responses (excellent, very good, good, fair, and poor). None of the participants from India or Switzerland perceived their health as "excellent." Of the 16 participants from India, one rated perceived health as "very good," nine rated their health as "good," five rated health as "fair," and one rated health as "poor" (Table 2). Six participants from Switzerland perceived their health as "very good," seven as "good" and only one participant rated health as "fair".

Nine participants from India had physical health scores in the low and medium ranges (0-75), and eight had mental health scores in the same range. One participant from Switzerland had low physical scores $(0-40)$, and five had medium physical health scores

Table 2 Self reported health of elders living in India and Switzerland

\begin{tabular}{lcc}
\hline Variable & India $(n=16)$ & Switzerland $(n=14)$ \\
\hline Age (mean + sd) & $74.8(7.6)$ & $70.4(6.3)$ \\
Gender (\% male) & $62.5 \%$ & $57.1 \%$ \\
Health status & & \\
Excellent & $0.0 \%$ & $0.0 \%$ \\
Very Good & $6.2 \%$ & $42.9 \%$ \\
Good & $56.3 \%$ & $50.0 \%$ \\
Fair & $31.3 \%$ & $7.1 \%$ \\
Poor & $6.2 \%$ & $0.0 \%$ \\
Physical functioning score -SF-12 & & \\
Low (0-40) & $37.5 \%$ & $7.1 \%$ \\
Medium (41-75) & $18.7 \%$ & $35.7 \%$ \\
High (76-100) & $43.8 \%$ & $57.2 \%$ \\
Physical functioning score (mean + sd) & $54.4(34.8)$ & $76.8(22.7)$ \\
Mental functioning score -SF-12 & & \\
Low (0-40) & $6.2 \%$ & $0.0 \%$ \\
Medium (41-75) & $43.8 \%$ & $28.6 \%$ \\
High (76-100) & $50.0 \%$ & $71.4 \%$ \\
Mean mental functioning (mean + sd) & $72.1(20.5)$ & $83.6(11.5)$ \\
ADL (\% able) & $75.0 \%$ & $100 \%$ \\
IADL (\% able) & $68.7 \%$ & $92.8 \%$ \\
\hline
\end{tabular}


(41-75). Four participants from Switzerland had medium mental health scores (41-75), while ten had high mental health scores (76-100). Percent and mean physical and mental health scores are presented in Table 2.

Among the participants from India, 11 had no problem managing either their ADLs or IADLs (Table 2). One had problems with IADLs only, while the remaining four faced challenges in performing both ADLs and IADLs. All participants from Switzerland were able to address their basic functional needs; only one could not perform all IADLs.

Personal factors affecting health

Participants from both India and Switzerland acknowledged that personal factors such as proper nutrition, open-mindedness, and cleanliness were critical for maintaining their health. Only elders who participated from Switzerland felt that actively seeking positive health behaviors was an important factor for good health. These positive health seeking behaviors included activities such as walking, biking, and accessing available healthcare resources.

Nutrition Following the right diet and ensuring proper intake of nutrients was one practice that all participants considered important for good health. Interestingly, they understood that their eating habits should be different from those practiced while growing up in Tibet. Staple foods for Tibetans living in Tibet are tsampa (roasted barley) and meat.

Living in diaspora, the participants understood that eating a balanced diet that included green vegetables and low amounts of salt, red meat, and oil improved their health. Participants in Switzerland consumed a more balanced diet than those in India because over time they had acquired Swiss eating habits. The ability of participants living in India to consume healthy food correlated to their ability to afford it. Mingmar, a man aged 75, said, "I [try to] eat well, but buying good food depends on my financial circumstances. I am able to consume good food when I have money."

Open-mindedness Open-mindedness was also deemed vital for good health because it enabled them to think more freely and face challenges with as little stress as possible. In Tibetan language, open-mindedness (sem lho thongya chenpo) incorporates within it the ability to think and analyze a situation with a holistic view, so that, when a person encounters a problem, he or she could broadly evaluate it and not be overwhelmed. Tashi, a man aged 64, expressed this belief, "Open mindedness is good for health because openmindedness helps one to solve problems and face challenges with ease. It allows one to be calm and happy."

Cleanliness Public health notions of cleanliness, such as washing hands and groceries before consumption, keeping food fresh, maintaining a sanitary environment, and keeping oneself clean were relatively new concepts for these Tibetan elders. Participants recalled that maintaining their present level of cleanliness was not required in Tibet because of its high altitude and cold weather, which kept food consumable for long periods of time. Also, because of the cold Tibetan climate, it was not necessary to wash oneself everyday or even frequently. Nevertheless, they realized that they could no longer continue their Tibetan habits in India or Switzerland. By necessity, they had developed new habits. 
Connection with family

Of the 30 elders who participated in this study, six had no children and the other 24 had one or more children (Table 1). Children of participants living in India tended to live far away from their parents. Half with children had at least one child who lived in the West. However, ten participants also had a child either living at home or in neighboring Tibetan communities in the Bylakuppe settlement, typically only an hour away by bus. In contrast, the children of participants living in Switzerland mostly lived in the same canton or in neighboring cantons. Most children were no more than an hour's distance away from their parents.

Married participants were living with their spouse, and one never married and one widowed participant reported living with a relative. The participants reported positive emotional benefits associated with having children, a spouse, or relatives. Their children provided help when needed, and the elders perceived them as a source of support in the event of future needs. Participants stressed that they needed to remain healthy as long as possible, as good health was beneficial to both them and their children.

Only two participants, Yangchen, a woman aged 63 from Switzerland, and Lhakpa, a man aged 65 from India, felt that there was no correlation between one's family and health. Yangchen stated, "Parents and children have no contribution to one's health. Health is dependent on oneself. I do not share my problems with my children because it just increases the problem when they start worrying about you."

\section{Community}

Participants in India were living in a Tibetan settlement where their neighbors were Tibetans, while those in Switzerland were living in the local community among Swiss people but within close proximity to other Tibetan families. For all participants, the Tibetan community provided a continuing sense of happiness as it allowed them to continue observing Tibetan traditions and culture in the host nation. The community enabled an environment where they can practice their Tibetan religion both individually and as a community. Because everyone shared a common past, they could talk with other Tibetans without language or cultural barriers which also increased overall satisfaction.

A never married man, Tashi, aged 64, articulated the support he received from his community,

Tibetans in this community will help [me] in case of need because here people help one another. I believe that Tibetans will help, and I will be fine. It is beneficial to live in Tibetan community. There would be problem if I lived in Indian community. I would not be happy. I have good emotional well-being because I live in Tibetan community. In seeing a Tibetan, there is a feeling of happiness, and there is happiness being with your own people.

As evidenced in the previous quote, in addition to the support received from the community, simply living in a Tibetan community was beneficial to emotional health. Particularly in India, the participants reported that the community provided essential support to needy older adults. These support included ensuring that older adult received regular meals and medical care.

\section{Healthcare}

All participants felt that availability of healthcare resources were also critical for good health. Elders from both India and Switzerland agreed that healthcare facilities lacked in 
Tibet (i.e., pre-1959 Tibet). The study participants stated that in pre-1959 Tibet, there were very few Tibetan medical doctors, and these few were rarely accessible in rural regions. Only wealthy families could afford the transportation and treatment cost of a physician. For nearly any illness in Tibet, they depended largely on homemade remedies and prayers. Goldstein et al. (2010) report the availability of health care access in Tibet today.

Healthcare access in India Participants' perception of their health in India varied. Half of the participants thought that their health would be better if they were still in Tibet. They reported that in Tibet they had never heard of the ailments about which they now knew and from which some suffered. Ngodup, a man aged 77, stated that Tibetans did not have high blood pressure in Tibet because of its good water and air, but in India, he and many others suffered from hypertension.

The remaining half of the participants felt that their health was better in India due to their increased access to healthcare resources. These resources included the exile Tibetan government Department of Health's western medicine based medical hospital and Tibetan medicine clinics in Bylakuppe. In India, such medical facilities are available in different Tibetan settlements and its aims are to provide accessible healthcare centers to address the physical and mental health needs of people living there. In addition to medical facilities provided in the settlement, extensive healthcare facilities are available in Indian towns and cities close to the settlement. Availability of such resources ensured that the participants could receive a diagnosis for their illness easily and gain timely treatment.

Regardless of perceptions of health in India as compared to pre-1959 Tibet, 12 of the 16 participants reported suffering from and seeking medical help for at least two health conditions. The most common ailments were poor eyesight, knee pain, and hypertension, followed by back pain, indigestion, ear problems, shoulder pain, asthma, headaches, and cough.

The healthcare utilized by most participants consisted of traditional Tibetan medicines available through the local Tibetan medical clinic. A Tibetan physician made monthly visits to the community in order to provide medical services. All participants sought medical care from this physician regularly because it was cheaper than visiting other healthcare centers, and they could easily access the facility on foot.

Some participants also used western medical care provided in the main settlement, neighboring Indian towns, and major cities. However, the elders reported that they would go to the hospital when they needed more specialized attention than the regular Tibetan physician could provide. Upon the recommendation of local doctors from the settlement hospital, larger hospitals in the cities were consulted when needed.

Many participants preferred not to seek treatment from the larger and more specialized hospitals located in the cities because of negative attitude towards accessing healthcare there. Two participants doubted the efficiency of hospitals in the cities, stating that hospitals were simply in the business of earning money. They believed that doctors kept patients in the hospital only as long as the patients could pay and then discharged them.

Furthermore, seeking treatment from hospitals in the cities meant a greater financial burden on elders' already limited resources. Penpa, a woman aged 79, who had a broken wrist, stated that the doctor recommended surgery to fix it, which would cost her approximately 100,000 rupees (equivalent to $\$ 2,500$ ). She and her husband decided against the surgery because they were living on limited financial support from their daughter, and they did not want to overburden her.

Transportation to healthcare facilities was available, but some participants faced challenges in accessing these services. Many participants suffered from knee pain, which made it difficult for them to travel long distances without assistance. The few who were 
relatively healthy rode motorbikes, but for the others, traveling was difficult. Other options consisted of taking transportation such as buses and taxis. Despite knowing how to access transportation, most elders were not comfortable traveling alone. Finding a seat on the bus was often difficult, and the hot climate made traveling arduous.

Healthcare access in Switzerland All participants from Switzerland perceived that their health was better there because of the availability and accessibility of healthcare services. They had health insurance, compulsory in Switzerland for all residents. They stated that healthcare access had never been a problem for them. They had family doctors who they visited, and their physician admitted them to the hospital if necessary. A few participants reported that they would already be dead in India or Tibet because of the lack of hygiene and healthcare resources, indicating that their longer life span was due to the availability of better resources in Switzerland. Namgyal, a man aged 67, believed the health of his generation to be significantly better than that of previous generations.

Healthcare facilities are better here, and people have financial security. There is good and equal access to healthcare in Switzerland. In [pre-1959] Tibet, healthcare access was only available to individuals who were from rich families. In Switzerland, I have seen people get better with bad diseases and have seen people live longer because of good facilities. Here, doctors have patients' histories, and they provide good treatment. Also, our health is better than our parents' because healthcare system was different [unknown] in [pre-1959] Tibet.

Participants were asked to list the health conditions from which they were suffering to understand their healthcare usage. Only two of 14 participants stated that they were healthy and did not have any health problems. The other 12 were suffering from one or more ailments, including knee pain and hypertension, followed by shoulder pain, kidney problems, heart disease, back pain, arthritis, and ear and eye problems.

The participants sought medical treatment when needed. Neither finances nor transportation was an issue. Yeshi, a man aged 72, suffered from lung fibrosis and received medical treatment six to seven times a year. Tsewang, a man aged 62, had severe back and shoulder pain. He required regular interventions to control his pain which was severe at times. Other participants did not have regular or yearly physical checkups because they felt that check-ups were unnecessary.

\section{Religion}

Religion was omnipresent in the lives of all participants. They practiced religious activities on a daily basis, including making water offerings in the morning, prostrating, and reciting prayers. In both countries, participants had a separate room or a special place in their home devoted to religious activities.

The participants believed that religion benefited them in this life and the next. Religion helped them understand karma, a belief that allowed them to accept their present circumstances and to understand the importance of their deeds, which would have an effect on their present life and death, as well as the next life. They assumed that prayers prevented unfortunate incidences such as illness. Many believed that engaging in religious activities was just as important for their health as practicing healthy behaviors. Gyaltsen, a man aged 92, stressed, "Religion and culture have positive influence on health because prayers prevent illness. Also, prayer brings prosperity. Thus, for good health, timely consultations with doctors and prayer are [both] necessary." 
Study participants also stated that religion had a positive effect on their health. This positive impact was described as emotional well-being realized by regular recitation of prayers and fulfillment of daily religious activities. They felt content when they performed such activities regularly. In addition to improved emotional health, those in India received a benefit in the form of improved physical health. The participants in India regularly engaged in numerous circumambulations at the small temple in their village which increased their physical activity.

\section{Government policies}

Perceptions of participants in India and Switzerland varied the most regarding the impact of government policies on health. In India, all older Tibetans do not receive pensions or social benefits, neither from the Indian government nor from the Tibetan exile government. Only retired Tibetans who have worked for the exile government are entitled to a pension. The exile government has put in place some security net for poor older Tibetans. These include old people's homes for elders who have no children or relatives to care for them, and some stipend and/or sponsors for elders who need financial help. One such old people's home is located in the Bylakuppe settlement. No participant expressed a desire to go to the old people's home, which they viewed as a last resort only. One participant, Gyalpo, a man aged 76, without any children, reported receiving a small financial support from a sponsor.

In contrast, all of the participants in Switzerland praised the policies of the Swiss government, which enabled them to maintain an income level that ensured their financial independence and good health. They received both social security and pensions. Topgyal, a man aged 72, stated, "Switzerland has good policies such as old age security for older adults. My old age is secured. I worked until retirement, and now I get old age benefits and pension."

They recognized these policies as good government planning that ensured their safety and autonomy into old age. Sonam, a man aged 79, emphasized, "Government help is good for health. If one does not have money, government provides for insurance and other benefits. You do not have to ask for help, you get it."

\section{Discussion}

Most elders who participated in this research were functionally healthy and could perform basic and instrumental activities of daily living. Participants living in India had more problems associated with ADLs and IADLs than did those in Switzerland. They also reported poorer perceived health and had lower physical and mental health scores than those living in Switzerland. This may be a result of the more difficult lives that those in India experienced and the considerable systemic benefits that those in Switzerland received.

The majority of the participants had good mental health scores, which were better than their physical health scores. Research studies on refugees have documented the presence of depression and PTSD, even many years after traumatic experiences (Bean et al. 2007; Marshall et al. 2005). Similar to other refugee groups, Tibetan refugees in India also suffer from depression and PTSD (Holtz 1998; Mills et al. 2005). The presence of depression and PTSD in the present sample was not examined because they migrated five decades earlier. Depression and PTSD related to their traumatic experiences could not be validated easily. In addition, Tibetans received structural support from the exile and host governments, which eased their lives and enabled them to live as a community in a foreign land, one in 
which they were able to preserve some sense of cohesiveness. Tibetan refugees have gained considerable economic success in exile (Mountcastle 1997; von Welck and Bernstorff 2004) which may have eased their emotional distress. The Tibetan Buddhist beliefs may also play a role in the acceptance of adults' traumatic past and in other difficult situations that they face.

Most participants were suffering from one or more ailments. The most common were hypertension, knee pain, back pain, and ear and eye problems. The health problems that the participants reported is consistent with research reporting high prevalence of hypertension, knee pain, and back pain among older Tibetans (Bera 2004; Bhatia et al. 2002; Tripathy et al. 2006). These studies also found that elder Tibetans suffer from obesity and cardiovascular disease, mimicking the disease burden of developed countries. However, only one elder reported heart disease, and none suffered from obesity. This group of elders may be healthier than those sampled by other studies.

\section{Microsystem}

Participants from both India and Switzerland have adapted to the new public health standards of diet and cleanliness. Reports on health adaptation of Tibetans in India concluded that Tibetans continue to consume higher levels of protein than is generally recommended (Bera 2004; Planning Commission 2004). The participants understood that their eating habits should be different from those maintained while growing up in Tibet, and they knew the importance of consuming a balanced diet consisting of more green vegetables, less salt, red meat, and oil. However, affordability was an issue, particularly for some in India. Barnes and Almasy (2005) and Pieterse and Ismail (2003) also reported that refugees had good understanding of healthy behaviors, though they failed to follow them because of money and time constraints.

\section{Mesosystem}

Similar to studies that reveal the positive effect of social networks on individual health and well-being (Ajrouch 2007; Childs et al. 2011; Diwan et al. 2004), these Tibetan elders reported positive emotional benefits associated with having a spouse, children, parents, and/ or other relatives nearby. Those with a spouse had someone with whom they could share their old age. Moreover, participants in India resided in Tibetan settlements and those in Switzerland lived in close proximity to other Tibetan families. Both groups stated that their community provided them security and happiness. Living in a co-ethnic community provided the refugees with much-needed social support and buffered pressures to assimilate into the host nation (Esses et al. 2001; Reitz and Sklar 1997). Further the refugees could bond and share their experiences with others with the same beliefs and values (Chaaya et al. 2007; Litwin 2006).

\section{Exosystem}

The study participants agreed that the availability of healthcare resources was an important factor for good health. For individuals to have good health, accessible, affordable, and efficient healthcare service is critical (Fung and Wong 2007; Wong et al. 2006). They experienced negligible healthcare access in pre-1959 Tibet and moderate healthcare services in India. Healthcare insurance was virtually unknown to them. Their access to healthcare is dependent on their ability to pay for services. Those in Switzerland enjoyed a 
very high standard of healthcare resources due to the universal healthcare system (Schoenenberger and Stuck 2006).

Healthcare utilization varied between elders from India and Switzerland and was dependent upon severity of illness and cost. A study on social support received by older parents from externally living daughters in Tibet found that older parents were hesitant in seeking medical treatment when it was expensive (Childs et al. 2011). In addition, for participants residing in India, the first healthcare option was consultation with the traditional Tibetan doctor in the local village. This was so because it was cheaper than visiting other healthcare centers, and the elders could walk to the clinic. They also preferred it because of their familiarity with Tibetan medicine (Bera 2004).

The participants' second choice was the health center at the Bylakuppe Settlement, which they consulted when their pain could not be managed by traditional medicine. This health service is accessible through public transportation, and the medical costs are subsidized by the Tibetan exile government. Finally, only upon the recommendation of doctors from the Bylakuppe health center would the elders consult hospitals in larger cities such as Mysore. They hesitated to seek healthcare in the cities because it symbolized the severity of their illness. The finding is in line with other studies that highlighted distrust as a factor for poor health seeking behaviors among minorities (Benisovich and King 2003; Feldman et al. 2007; Kosa et al. 2007), participants distrusted the hospitals and chose not to access healthcare.

Very different from participants living in India, the participants in Switzerland had access to healthcare services. Availability of health insurance mitigated the problem of affordability. Seeking medical treatment was never a problem for them, and so they sought medical treatment as needed. Transportation was also not an issue due to the availability of efficient public and emergency transportations.

\section{Macrosystem}

Two macro-level factors that affected the health of the elders were religion and government policies. The positive effects of religion on health and well-being have been well established in research on aging (Braam et al. 2008; Krause et al. 1999; Koenig 2007; Krause 2006). Studies have found that among immigrants, religious activity is associated with positive emotional well-being, lower mortality, and better physical health (Hahn et al. 2004; Yeager et al. 2006).

Religious activity was critical in the participants' lives. Literature concerning Tibetans living in diaspora have reported emotional satisfaction and happiness associated with completion of religious activities (Corlin 1991; Ott-Marti 1976; Wangmo and Teaster 2010). All participants emphasized the positive effect of religion on their present and next lives. They spoke of the positive impact of religion on their well-being. They believed that prayers brought good luck for them and their families. Importantly, the Tibetan religion bonded them to their community, thereby increasing their social support and improving their emotional well-being. The significance of religious activity for older Tibetans was also noted by Childs et al. (2011). They concluded that religious activities such as pilgrimage not only helped older parents prepare for their death and next life, but it also provided positive affect such as happiness because they had the chance to engage in social activities with non-kin peers.

Finally, government initiatives such as the provision of social security and pensions were beneficial in allowing elders to remain independent (Ahmadi and Tornstam 1996; de Valk and Schans 2008). Participants living in India and Switzerland varied in their perceptions of the effects of government policies on their health. In India, the participants did not receive pensions, either from the Indian government or from the Tibetan exile government. By 
contrast, all of the participants living in Switzerland lauded the policies of the Swiss government that enabled them to maintain an income level that ensured their financial independence and good health (Bolzman 2004; Schoenenberger and Stuck 2006). They received social security income from the government and pensions from their former employers as a part of their old age benefits and because of their life-long financial contributions into the Swiss system. They recognized these policies helping to ensure safety and autonomy in old age.

\section{Conclusions}

Regardless of challenges associated with migrating from one country to another and reestablishing economic, social, and cultural stability in the host nation, the elder Tibetan participants were able to maintain their health and well-being in diaspora. Most participants were healthy and functionally competent. The elders, who participated from Switzerland, were healthier than those from India. Participants adapted to healthcare behaviors of their host nation by incorporating learned behaviors into their daily lives.

Having social networks of support from the family and the community were important factors that affected the health and well-being of the participants. Living in a Tibetan community and in close proximity to other Tibetans fostered a sense of belongingness and well-being for the participants. The community was also an important source of support, particularly for those without children. Government policies of old age benefits such as social security, pensions, and healthcare insurance allowed older adults in Switzerland to enjoy a financially independent and secure old age. For those in India, lack of old age benefits restricted their healthcare access, as it was dependent upon their ability to pay for services and to trust the institution.

The study presents the important role played by different ecological systems (personal behaviors, family, community, religion, and government policy) in enabling the perceived health and health behaviors of elder Tibetans in their respective communities. Public health practitioners, social service providers, and other agencies involved with rehabilitation of refugees and immigrants must consider the impact of such factors in improving the health of these individuals and accelerating their adoption of positive health behaviors. Such parties could aid by providing immigrants and refugees with the knowledge of proper healthcare behaviors, the means of accessing healthcare, and capitalizing on available resources from the family and the ethnic culture.

Most importantly, the study provides a comparative analysis of systems, thereby presenting the crucial role played by old age benefits such as social security, pensions, and healthcare insurance in securing an independent old age of elder participants in Switzerland. Such old age benefits are increasingly becoming necessary for older refugees and other elders living in developing countries where such benefits are lacking and where elders are left behind as children migrate to urban centers or to other countries. The better health and well-being of older Tibetan participants living in Switzerland clearly presents a case for such old age benefits in developing countries for all older persons in general and older refugees in particular.

Acknowledgements I am grateful to the 30 Tibetan elders who shared their life experiences and opinions with me. This paper presents part of the data collected for my dissertation study and therefore, I thank my dissertation committee members, Dr. Pamela B. Teaster, Dr. Graham D. Rowles, Dr. Nancy Schoenberg, Dr. Joy Jacobs-Lawson, and Dr. Peggy Hickman, for their guidance and expertise. Finally, the positive and valuable suggestions provided by the two anonymous reviewers and the editor of this journal are highly appreciated. Their suggestions have tremendously aided in improving the quality of this manuscript. 


\section{References}

Adams, K. M., Gardiner, L. D., \& Assefi, N. (2004). Healthcare challenges from the developing world: Postimmigration refugee medicine. $B M J, 328,1548-1552$.

Ahmadi, F., \& Tornstam, L. (1996). The old flying Dutchman; shuttling immigrants with double assets. Journal of Aging and Identity, 3, 191-210.

Ajrouch, K. J. (2007). Resources and well-being among Arab-American elders. Journal of Cross-Cultural Gerontology, 22, 167-182.

Anand, D. (2000). (Re)imagining nationalism: Identity and representation in the Tibetan diaspora of South Asia. Contemporary South Asia, 9, 271-287.

Barnes, D. M., \& Almasy, N. (2005). Refugees' perceptions of healthy behaviors. Journal of Immigrant Health, 7, 185-193.

Bean, T. M., Eurelings-Bontekoe, E., \& Spinhoven, P. (2007). Course and predictors of mental health of unaccompanied minors in the Netherlands: 1 year follow -up. Social Science \& Medicine, 64, 1204-1215.

Benisovich, S. V., \& King, A. C. (2003). Meaning and knowledge of health among older adult immigrants from Russia: A phenomenological study. Health Education Research, 18, 135-144.

Bera, S. (2004). Tibetan refugees in India: Observations in the field of health. Journal of the Indian Anthropological Society, 39, 183-194.

Bernard, H. R. (2002). Research methods in anthropology: Qualitative and quantitative approaches (3rd ed.). Altamira: Walnut Creek.

Bhatia, S., Dranyi, T., \& Rowley, D. (2002). A social and demographic study of Tibetan refugees in India. Social Science \& Medicine, 54, 411-422.

Bolzman, C. (2004). Older labour migrants' well being in Europe: The case of Switzerland. Ageing and Society, 24, 411-429.

Braam, A. W., Schaap-Jonker, H., Mooi, B., De Ritter, D., Beekman, A., \& Deeg, D. (2008). God image and mood in old age: Results from a community-based pilot study in the Netherlands. Mental Health, Religion, and Culture, 11, 221-237.

Bronfenbrenner, U. (1977). Toward an experimental ecology of human development. American Psychologist, $32,513-531$.

Bronfenbrenner, U., \& Morris, P. A. (1998). The ecology of developmental processes. In W. Damon \& R. Lerner (Eds.), Handbook of child psychology: Vol. 1. Theoretical models of human development (5th ed., pp. 993-1028). New York: Wiley.

Burgess, A. (2004). Health challenges for refugees and immigrants. Refugee Reports, 25, 1-20.

Chaaya, M., Sibai, A. M., Fayad, R., \& El-Roueiheb, Z. (2007). Religiosity and depression in older people: Evidence from underprivileged refugee and non-refugee communities in Lebanon. Aging \& Mental Health, 11, 37-44.

Chakraborti, R. D. (2004). The graying of India: Population ageing in the context of Asia. New Delhi: Sage Publications.

Childs, G., Goldstein, M. C., \& Wangdi, P. (2011). Externally-resident daughters, social capital, and support for the elderly in rural Tibet. Journal of Cross-Cultural Gerontology, 26, 1-22.

Commission, P. (2004). Tibetan community in exile: Demographic and socio-economic issues 1998-2001. Dharamsala: Central Tibetan Administration.

Corlin, C. (1991). Chaos, order and world view: Tibetan refugees in Switzerland. Disasters, 15, $108-116$.

Cresswell, J. W. (2003). Research design: Qualitative, quantitative, and mixed methods approaches. Thousand Oaks: Sage Publications.

De Valk, H. A. G., \& Schans, D. (2008). 'They out to do this for their parents': Perceptions of filial obligations among immigrant and Dutch older people. Ageing \& Society, 28, 49-66.

Denzin, N. K., \& Lincoln, Y. S. (2000). The discipline and practice of qualitative research. In N. K. Denzin \& Y. S. Lincoln (Eds.), Handbook of qualitative research (2nd ed., pp. 1-28). Thousand Oaks: Sage.

Diwan, S., Jonnalagadda, S. S., \& Balaswamy, S. (2004). Resources predicting positive and negative affect during the experience of stress: A study of older Asian Indian immigrants in the United States. Gerontologist, 44, 605-614.

Dolma, S., Singh, S., Lohfeld, L., Orbinski, J. J., \& Mills, E. J. (2006). Dangerous journeys: Documenting the experience of Tibetan refugees. American Journal of Public Health, 96, 2061-2064.

Esses, V. M., Dovidio, J. F., Jackson, L. M., \& Armstrong, T. L. (2001). The immigration dilemma: The role of perceived group competition, ethnic prejudice, and national identity. Journal of Social Issues, 57, $389-412$.

Federal Stattistics Office. (2011). Population size and population composition-Data, indicators. Neuchàtel, Switzerland. Retrieved August 12, 2011 from http://www.bfs.admin.ch/bfs/portal/en/index/themen/01/ 02/blank/key/alter/gesamt.html. 
Feldman, C. T., Bensing, J. M., de Ruijter, A., \& Boeije, H. R. (2007). Afghan refugees and their general practitioners in the Netherlands: To trust or not to trust? Sociology of Health \& Illness, 29, 515-535.

Fung, K., \& Wong, Y.-K. R. (2007). Factors influencing attitudes towards seeking professional help among East and South East Asian immigrant and refugee women. The International Journal of Social Psychiatry, 53, 216-231.

Goldstein, M. C., \& Beall, C. M. (1997). Growing old in Tibet: Tradition, family, and change. Aging Asian Concepts and Experiences Past and Present, 643, 155-176.

Goldstein, M. C., Childs, G., \& Wangdui, P. (2010). Beijing's "people first" development initiative for the Tibet Autonomous region's rural sector-A case study from the Shigatse area. The China Journal, 63, 58-75.

Hahn, C.-Y., Yang, M.-S., Shih, C.-H., \& Lo, H.-Y. (2004). Religious attendance and depressive symptoms among community swelling elderly in Taiwan. International Journal of Geriatric Psychiatry, 19, 11481154.

Holtz, T. H. (1998). Refugee trauma versus torture trauma: A retrospective controlled cohort study of Tibetan refugees. The Journal of Nervous and Mental Disease, 186, 24-34.

Houston, S., \& Wright, R. (2003). Making and remaking Tibetan diasporic identities. Social and Cultural Geography, 4, 217-232.

Jamuna, D. (2000). Ageing in India: Some key issues. Ageing International, 16-32.

Joeffe, C., Brodaty, H., Luscombe, G., \& Ehrlich, F. (2003). The Sydney Holocaust study: Posttraumatic stress disorder and other psychosocial morbidity in an aged community sample. Journal of Traumatic Stress, 16, 39-47.

Johnson, J. M. (2002). In-depth interviewing. In J. F. Gubrium \& J. A. Holstein (Eds.), Handbook of interview research (pp. 103-119). Thousand Oaks: Sage.

Keyes, E. F., \& Kane, C. F. (2004). Belonging and adapting: Mental health of Bosnian refugees living in the United States. Issues in Mental Health Nursing, 25, 809-831.

Kleiger, P. C. (1992). Tibetan nationalism: The role of patronage in the accomplishment of a national identity. Meerut: Archana Publications.

Koenig, H. G. (2007). Religion and depression in older medical patients. American Association for Geriatric Psychiatry, 15, 282-291.

Korom, F. J. (1999). Tibetans in exile: A Euro-American Perspective. Journal of Transnational and Transcultural Studies, 1, 1-23.

Kosa, Z., Szeles, G., Kardos, L., Kosa, K., Nemeth, R., Orszagh, S., et al. (2007). A comparative health survey of the inhabitants of Roma settlements in Hungary. American Journal of Public Health, 97, 853-859.

Krause, N. (2006). Church-based social support and mortality. Journal of Gerontology: Social Sciences, 61B, S147-S152.

Krause, N., Ingersoll-Dayton, B., Liang, J., \& Sagisawa, H. (1999). Religion, social support, and health among the Japanese elderly. Journal of Health and Social Behavior, 40, 405-421.

Lawton, M. P., \& Brody, E. M. (1969). Assessment of older people: Self-maintaining and instrumental activities of daily living. Gerontologist, 9, 179-182.

Lee, Y.-A., Munsiff, S. S., Li, J., Driver, C. R., Mathema, B., \& Kreiswirth, B. N. (2001). Rising number of tuberculosis cases among Tibetans in New York city. Journal of Immigrant Health, 3, 73-180.

Litwin, M. S. (2002). RAND 12-Item health survey v2 (SF-12 v2) and UCLA prostate cancer index: Scoring instruction. Retrieved June 21, 2008 from http://www.uclaurology.com/site_uo/pdf/pci_long_scoring.pdf.

Litwin, H. (2006). Social networks and self-rated health: A cross-cultural examination among older Israelis. Journal of Aging and Health, 18, 335-358.

Marras, T. K., Wilson, J., Wang, E. E. L., Avendano, M., \& Yang, J. W. (2003). Tuberculosis among Tibetan refugee claimants in Toronto: 1998-2000. Chest, 124, 915-921.

Marshall, G. N., Schell, T. L., Elliot, M. C., Berthold, S. M., \& Chun, C.-A. (2005). Mental health of Cambodian refugees 2 decades after resettlement in the United States. Journal of the American Medical Association, 294, 571-579.

Mills, E. J., Singh, S., Holtz, T. H., Chase, R. M., Dolma, S., Santa-Barbara, J., et al. (2005). Prevalence of mental disorders and torture among Tibetan refugees: A systematic review. BMC International Health and Human Rights, 5, 7.

Mountcastle, A. (1997). Reframing refugees: The power of Tibetan identity. Collegium Anthropologicum, 21, 585-593.

Nelson, L. J., Naik, Y., Tsering, K., \& Cegielski, J. P. (2005). Population-based risk factors for tuberculosis and adverse outcomes among Tibetan refugees in India, 1994-1996. The International Journal of Tuberculosis and Lung Disease, 9, 1018-1026.

Ott-Marti, A. (1976). Problems of Tibetan integration in Switzerland. Ethnologia Europaea, 9, 43-52.

Pieterse, S., \& Ismail, S. (2003). Nutritional risk factors for older refugees. Disasters, 27, 16-36.

Prost, A. (2008). Contagion and its guises: Inequalities and disease among Tibetan exiles in India. International Migration, 46, 55-70. 
Reitz, J. G., \& Sklar, S. M. (1997). Culture, race, and the economic assimilation of immigrants. Sociological Forum, 12, 233-277.

Resnick, B., \& Nahm, E. S. (2001). Reliability and validity testing of the revised 12-item short-form health survey in older adults. Journal of Nursing Measurement, 9, 151-161.

Routray, B. P. (2007). Tibetan refugees in India: Religious identity and the forces of modernity. Refugee Survey Quarterly, 26, 79-90.

Schoenenberger, A. W., \& Stuck, A. E. (2006). Health care for older persons in Switzerland: A country profile. Journal of American Geriatrics Society, 54, 986-990.

Smith, M. (2003). Health care for refugees. Asia Pacific Family Medicine, 2, 71-73.

Soonthornchaiya, R., \& Dancy, B. L. (2006). Perceptions of depression among elderly Thai immigrants. Issues in Mental Health Nursing, 27, 681-698.

Strauss, A., \& Corbin, J. (1990). Basics of qualitative research: Grounded theory procedures and techniques. Newbury Park: Sage Publications.

Tripathy, V., \& Gupta, R. (2005). Birth weight among Tibetans at different attitudes in India: Are Tibetans better protected from IUGR? American Journal of Human Biology, 17, 442-450.

Tripathy, V., Satapathy, K. C., \& Gupta, R. (2006). Nutritional status and hypertension among Tibetan adults in India. Human Ecology, 14, 77-82.

von Welck, H., \& Bernstorff, D. (2004). Exile as challenge: The Tibetan Diaspora. Hyderabad: Orient Longman.

Wangmo, T. (2010). Changing expectations of care among older Tibetans living in India and Switzerland. Ageing and Society, 30, 879-896.

Wangmo, T., \& Teaster, P. B. (2010). The bridge from then to now: Tibetan elders living in diaspora. Journal of Applied Gerontology, 29, 434-454.

Ware, J. E., Kosinski, M., \& Keller, S. D. (1996). A 12-item short-form health survey: Construction of scales and preliminary tests of reliability and validity. Medical Care, 34, 220-233.

Wong, E. C., Marshall, G. N., Schell, T. L., Elliot, M. C., Hambarsoomians, K., Chun, C.-A., et al. (2006). Barriers to Mental health care utilization for U.S. Cambodian refugees. Journal of Consulting and Clinical Psychology, 74, 1116-1120.

Woodcock, G. (1970). Tibetan refugees in a decade of exile. Pacific Affairs, 43, 410-420.

Wrobel, N. H., Farrag, M. F., \& Hymes, R. W. (2009). Acculturative stress and depression in an elderly Arabic sample. Journal of Cross-Cultural Gerontology, 24, 273-290.

Yeager, D. M., Glei, D. A., Au, M., Lin, H.-S., Sloan, R., \& Weinstein, M. (2006). Religious involvement and health outcomes among older persons in Taiwan. Social Science \& Medicine, 63, 2228-2241.

Yeung, G. T. Y., \& Fung, H. H. (2007). Social support and life satisfaction among Hong Kong Chinese older adults: family first? European Journal of Ageing, 4, 219-227. 